\title{
The uniform primality conjecture for elliptic curves
}

\author{
by \\ Graham Everest (Norwich), Patrick Ingram (Toronto), \\ Valéry Mahé (Norwich) and Shaun Stevens (Norwich)
}

1. Introduction. The Mersenne problem asks if infinitely many integers of the form $2^{n}-1$ are prime. More generally, if $a>b$ are positive coprime integers, one can ask if the sequence

$$
V_{n}=\left(\frac{a^{n}-b^{n}}{a-b}\right)_{n \geq 1}
$$

has infinitely many prime terms. The answer is negative if $a / b$ is a perfect power. For example, if $a=A^{2}$ and $b=B^{2}$, with $A, B \in \mathbb{Z}$, then the terms factorize as

$$
V_{n}=\left(\frac{A^{n}-B^{n}}{A-B}\right)\left(\frac{A^{n}+B^{n}}{A+B}\right)
$$

for $n$ odd and

$$
V_{n}=\left(\frac{A^{n}-B^{n}}{A^{2}-B^{2}}\right)\left(A^{n}+B^{n}\right)
$$

for $n$ even. It is easy to see that neither factor may be a unit when $n$ is large enough. Indeed, since the greatest common divisor of the two terms on the right (in either expression) is easily controlled, there are few possibilities for $V_{n}$ to be a prime power, a remark germane to this paper.

Let $E / \mathbb{Q}$ denote an elliptic curve, given by a Weierstrass equation with integer coefficients, and let $P \in E(\mathbb{Q})$ denote a non-torsion point. One can always write

$$
n P=\left(\frac{A_{n}}{B_{n}^{2}}, \frac{C_{n}}{B_{n}^{3}}\right)
$$

2000 Mathematics Subject Classification: 11G05, 11A41.

Key words and phrases: canonical height, divisibility sequence, division polynomial, elliptic curve, isogeny, prime, rational function field, reduction, Siegel's theorem.

The research of the second author was supported by grant from NSERC of Canada and one from the LMS. The research of the third author was supported by a grant from EPSRC. 
with $A_{n}, B_{n}$, and $C_{n} \in \mathbb{Z}$, and $\operatorname{gcd}\left(A_{n}, B_{n}\right)=1$. The sequence $\left(B_{n}\right)_{n \geq 1}$ is an elliptic divisibility sequence. These sequences have been the focus of attention of a number of authors: consult $[4,6,8,10,11,14,24,25,30$, $31,32,33,34]$. The role of an elliptic divisibility sequence relative to the addition law of $E(\mathbb{Q})$ is analogous to the role of the sequence (1) relative to the multiplicative group of $\mathbb{Q}$. It is unlikely that $B_{n}$ will ever be prime, because of the relation $B_{1} \mid B_{n}$. It is natural, as in the multiplicative case, to ask when $B_{n} / B_{1}$ might be prime, or a prime power.

1.1. Background. Chudnovsky and Chudnovsky [3] considered the question of prime occurrence in elliptic divisibility sequences computationally, finding some examples of prime values which are large (several hundred decimal digits) in comparison with the starting data. These computations were not easy to perform on several counts. For one thing, the terms of the sequences grow very rapidly - the logarithm is quadratic in the index, and proportional to the global canonical height. Also, there was a shortage of known small height points. Finally, the computing power at the time was much less than what is available today. With the examples to hand, searching was feasible only out to index around $n=100$. Much larger prime terms in elliptic divisibility sequences have been found recently.

Example 1.1. Consider

$$
E: y^{2}+y=x^{3}-x \quad \text { with } P=[0,0] \text {. }
$$

The following table shows indices $n$ for which $B_{n}$ is prime (actually, a MillerRabin pseudo-prime to 10 randomly chosen bases), together with the number $\# B_{n}$ of decimal digits of $B_{n}$ :

\begin{tabular}{ccccccccccccccc}
\hline$n$ & 5 & 7 & 8 & 9 & 11 & 12 & 13 & 19 & 23 & 29 & 83 & 101 & 409 & 1291 \\
$\# B_{n}$ & 1 & 1 & 1 & 1 & 2 & 2 & 2 & 4 & 6 & 10 & 77 & 114 & 1857 & 18498 \\
\hline
\end{tabular}

The final two primes were found by Bríd Ní Fhlathuín (1999) and the first author (2006) respectively using MAGMA [19] and PARI-GP [22]. The largest takes a couple of hours to test for (pseudo-)primality.

See also Example 4.2 for a large prime term found recently. Bearing in mind that all of the examples mentioned have appeared at the outer limits of what is computationally feasible at the time this might suggest that some elliptic divisibility sequences contain infinitely many prime terms. On the other hand, in [6], all of the examples from [3] were tested out to $n=500$ but no more prime terms appeared. Furthermore, while it is true that the primes in Example 1.1 are large, this sequence has, up to the present, produced only 14 prime terms. It also manifests a pattern witnessed in several examples (see the table in Section 4.2), namely, that a gap principle seems to be at work. What this means is that the gaps between prime 
terms grow quickly in proportion to the index. Taking this together with the quadratic exponential growth rate of the sequence forces any outlying prime term to be inordinately large. A gap principle has recently been articulated successfully in the study of integral multiples of a fixed integral point [15]. In Section 4.2, the results of extensive computations are exhibited, showing the appearance of prime terms in elliptic divisibility sequences generated by the 18 rational points with smallest known global canonical height in Elkies' table [7]. (These, together with all the computations performed for this paper, used both [19] and [22].) Together with a heuristic argument from [6], revisited in Section 4.1, this lends support to the following (see $[6,8,11])$ :

Conjecture 1.2 (Primality conjecture). If $B=\left(B_{n}\right)$ denotes an elliptic divisibility sequence generated by a rational point on an elliptic curve, then the number of prime terms $B_{n} / B_{1}$ is bounded.

In [11] it was demonstrated that if $P \in E(\mathbb{Q})$ lies in the image of a non-trivial isogeny then $B_{n}$ can be prime (indeed a prime power) for only finitely many $n$. When the isogeny is the endomorphism $[k]$ (multiplication by the integer $k$ ), this is in direct analogy with the trivial case for sequences of the form (1), discussed earlier. The proof works by giving a preliminary argument showing the existence of a canonical factorization, then estimates arising from a strong form of Siegel's theorem show that each factor is too large to be a unit.

In [8], these techniques were generalized and improved and a stronger form of Conjecture 1.2 appeared. This predicts a uniform bound on the number of prime power terms assuming the curve is in minimal form - the uniform primality conjecture. In the current article, a proof of that conjecture will be provided, assuming Lang's conjecture, in the case where descent via an isogeny is possible. The methods include the use of a quantitative version of Siegel's theorem proved by Silverman. In Section 4 a discussion is included about the plausibility of the uniform primality conjecture when descent is not possible. As the question entails the application of results from diophantine approximation, it is also interesting to consider the problem over the function field $\mathbb{Q}(T)$, where stronger diophantine approximation results are known (for example, Lang's conjecture). Stronger conclusions do indeed become possible.

1.2. Main results. Let $K$ be $\mathbb{Q}$ or $\mathbb{Q}(t)$, and let $\mathcal{O}_{K}$ be $\mathbb{Z}$ or $\mathbb{Q}[t]$ respectively. Let $E$ be an elliptic curve over $K$, in Weierstrass form, having coefficients in $\mathcal{O}_{K}$. Let $P \in E(K)$ and write

$$
x(P)=\frac{A_{P}}{B_{P}^{2}},
$$


with $A_{P}, B_{P} \in \mathcal{O}_{K}$ coprime and $B_{P}>0$ in the case $K=\mathbb{Q}$, or $B_{P}$ monic in the case $K=\mathbb{Q}(t)$.

Much of the discourse assumes a conjecture of Lang, which arises naturally, and appears to be a necessary assumption in any attempt to make uniform statements about the divisibility properties of elliptic divisibility sequences $[10,14,16,17]$. This conjecture will now be stated and relies upon definitions provided in full in Section 2.1. It is known that the canonical height $\widehat{h}(P)$ is zero if and only if $P$ is a point of finite order, so it is natural to ask how small $\widehat{h}(P)$ might be for non-torsion points $P$. This question turns out to be quite important: in general, quantitative estimates for diophantine approximation on elliptic curves all rely on some sort of lower bound on $\widehat{h}(P) / h(E)$, where $h(E)$ is the height of the curve. Considering elliptic curves in general, it is not hard to show that $\widehat{h}(P) / h(E)$ can be made arbitrarily small (without resorting to choosing torsion points $P$ ). For minimal curves $E / K$, however, this seems not to be the case.

LANG'S CONJECTURE. There exists $\delta>0$, which depends only on $K$, such that $\widehat{h}(P) \geq \delta \max (1, h(E))$ for all minimal curves $E / K$ and all nontorsion points $P \in E(K)$.

Our main results use a definition which appeared first in [11] and in a more general form in [8]. In [11], some crude counting amongst small conductor curves suggested that when $K=\mathbb{Q}$, the definition applies in a very large number of non-trivial cases.

Definition 1.3. If $P \in E(K)$ is non-torsion and is the image of a $K$-rational point under a non-trivial $K$-rational isogeny, then say that $P$ is magnified.

By composing the pre-image of the isogeny with an isomorphism if necessary, it may always be arranged that the pre-image is in minimal form. This will be a standing assumption throughout the paper.

In the rational case, the main theorem of the paper is as follows (see Theorem 2.1 for a more precise version):

Theorem 1.4. Assume $K=\mathbb{Q}$ and $B$ is an elliptic divisibility sequence generated by a magnified $K$-rational point on an elliptic curve in minimal form. If Lang's conjecture holds then the number of prime power terms $B_{n} / B_{1}$ is bounded independently of the curve, the point and the degree of the isogeny.

When $K=\mathbb{Q}(t)$, as anticipated, an unconditional version of Theorem 1.4 holds. A bigger surprise is the different character of the main conclusion.

TheOrem 1.5. Assume $K=\mathbb{Q}(t)$ and $B$ is an elliptic divisibility sequence arising from a magnified $K$-rational point on an elliptic curve 
in minimal form. Then $B_{n} / B_{1}$ fails to be a prime power for all indices $n \geq 4160366$.

The different nature of the two results is worthy of comment and Sections 3 and 4 are given over to a full discussion of the different cases. At this point, the following question seems worth asking, and it is one that we have not been able to resolve.

Question. In the case when $K=\mathbb{Q}$, assume that $B$ is an elliptic divisibility sequence generated by a magnified $K$-rational point on an elliptic curve in minimal form. If Lang's conjecture is true, does it follow that $B_{n} / B_{1}$ fails to be a prime power for all $n$ beyond some uniform bound?

A positive answer to this question is certainly desirable, because it means that a large class of explicit examples can, at least hypothetically, be tested to find all the prime terms. With current techniques, explicit examples do exist [9] (see also Example 4.1), but for a limited class of curves. Theoretically too, a positive answer is satisfying because it means that the two cases when $K=\mathbb{Q}$ and $K=\mathbb{Q}(t)$ are exactly parallel, assuming the generating point $P$ is magnified. Note however that, over $K=\mathbb{Q}(t)$, the primality conjecture itself is not likely to be true - a discussion, which includes some striking examples, follows in Section 3.

In Section 2, a proof will be given that, under Lang's conjecture, a uniform bound exists for the number of prime terms $B_{n} / B_{1}$ which applies in both the rational and the function field cases, assuming the elliptic divisibility sequence is generated by a magnified point on a minimal curve. This section highlights the convergence of the two theories. In Sections 3 and 4, various points of divergence will be discussed, often with reference to explicit examples.

\section{The uniform primality conjecture in both cases}

2.1. Notation. The following notation will be standard throughout:

$K$ either $\mathbb{Q}$ or $\mathbb{Q}(t)$;

$\mathcal{O}_{K} \quad$ either $\mathbb{Z}$ if $K=\mathbb{Q}$ or $\mathbb{Q}[t]$ if $K=\mathbb{Q}(t)$;

$v$ a prime of $K$ with completion $K_{v}$ and ring of integers $\mathcal{O}_{v}$;

$\log |\cdot|_{\infty}$ either $\log (|\cdot|)$ if $K=\mathbb{Q}$ or deg if $K=\mathbb{Q}(t)$;

$h(p / q)$ the height of $p / q$ defined, for $p, q \in \mathcal{O}_{K}$ coprime, by

$$
h(p / q):=\max \left(\log |p|_{\infty}, \log |q|_{\infty}\right) ;
$$

$E$ an elliptic curve over $K$;

$\Delta$ or $\Delta_{E}$ the discriminant of $E$; 
$j$ or $j_{E}$ the $j$-invariant of $E$;

$h(E)$ the height $h(E):=\frac{1}{12} \max (h(j), h(\Delta))$ of $E$;

$P$ a point of $E(K)$;

$h(P)$ the height $h(P):=\frac{1}{2} h(x(P))$ of $P$;

$\widehat{h}(P)$ the canonical height of $P$, defined by

$$
\widehat{h}(P):=\lim _{n \rightarrow \infty} \frac{h(n P)}{n^{2}} .
$$

Generally speaking, when the existence of a uniform constant is postulated, what is meant is a constant independent of the choice of the elliptic curve (and the point studied if there is one). Of course, such a constant may depend on the choice of the base field $K$.

2.2. Reduction to Siegel's theorem. For ease of exposition, define the Lang ratio of $P \in E(K)$ to be $\varrho(P, E)=\widehat{h}(P) / \max (1, h(E))$. Then Lang's conjecture says that there exists $\delta>0$ such that

$$
\varrho(P, E) \geq \delta
$$

for all minimal curves $E / K$ and all non-torsion points $P \in E(K)$. Silverman [28] has shown, for number fields $K$, that $\varrho(P, E)$ may be bounded below in terms of the number of primes at which $E / K$ has split multiplicative reduction. Expanding on these ideas, Hindry and Silverman [12] showed that $\varrho(P, E)$ may be bounded below in terms of an upper bound on the Szpiro ratio of $E / K$, that is, the ratio of the log-discriminant of $E$ to the log-conductor. Hindry and Silverman also showed that if $K$ is a onedimensional function field, then the Szpiro ratio of an $S$-minimal elliptic curve (where $S$ is a set of primes of $K$ ) is bounded above absolutely in terms of $S$; in particular, with $S=\{\infty\}$, Lang's conjecture holds. In this paper, it will often be convenient to work in terms of $\varrho(P, E)$ in order to obtain unconditional results.

The following is a more precise version of Theorem 1.4, valid both for $\mathbb{Q}$ and for $\mathbb{Q}(t)$.

THEOREM 2.1. For any $\delta>0$, there is a constant $M_{\delta}$ with the following property: Let $B=\left(B_{n}\right)$ be an elliptic divisibility sequence arising from a magnified $K$-rational point $P$ on an elliptic curve $E$ in minimal form. Write $\sigma: E^{\prime} \rightarrow E$ with $E^{\prime}$ in minimal form, and $\sigma\left(P^{\prime}\right)=P$. If $\varrho\left(P^{\prime}, E^{\prime}\right) \geq \delta$, then the number of prime power terms $B_{n} / B_{1}$ is bounded by $M_{\delta}$.

In light of the above-mentioned results of Hindry and Silverman, in the case $K=\mathbb{Q}(t)$, there is a uniform constant $\delta>0$ such that the condition $\varrho\left(E^{\prime}, P^{\prime}\right) \geq \delta$ is always satisfied. If Szpiro's conjecture holds, then this is true of $K=\mathbb{Q}$ as well.

Similar methods allow a result of the following kind: 
TheOREM 2.2. For any $\delta>0$, there is a constant $C_{\delta}$ with the following property: if $\phi: E^{\prime} \rightarrow E$ is an isogeny of minimal elliptic curves, then for any subgroup $\Gamma^{\prime} \subseteq E^{\prime}(\mathbb{Q})$ such that $\min _{P^{\prime} \in \Gamma^{\prime}} \varrho\left(E^{\prime}, P^{\prime}\right) \geq \delta$,

$$
\#\left\{P \in \phi\left(\Gamma^{\prime}\right): B_{P} \text { is a prime power }\right\} \leq C_{\delta}^{1+\operatorname{rank}\left(\Gamma^{\prime}\right)} .
$$

It follows immediately that the number of prime power terms in the sequence $\left(B_{n}\right)_{n \geq 1}$ is bounded uniformly. This observation is not nearly as strong as Theorem 2.1 unless one restricts attention to sequences in which $B_{1}=1$, which are in some sense rare (corresponding to integral points on elliptic curves).

2.3. Behaviour under isogeny. The first lemma below shows how primes behave under isogeny, and demonstrates that the denominators of points in the image of an isogeny admit a canonical factorization - see (6), in Sections 3.5.

Lemma 2.3. Let $E$ be a minimal elliptic curve defined over $K$, and let $\sigma: E^{\prime} \rightarrow E$ be an isogeny of degree $m$. Then we have

$$
v\left(B_{P}\right) \leq v\left(B_{\sigma(P)}\right) .
$$

If $E^{\prime}$ is also minimal, then $v\left(B_{P}\right)>0$ implies

$$
v\left(B_{\sigma(P)}\right) \leq v\left(B_{P}\right)+v(m) .
$$

Proof. On the assumption that $E^{\prime}$ is minimal at $v$, it is not hard to show (see, for example, the exposition in [33]) that the isogeny $\sigma$ induces a map of formal groups $F_{\sigma}: \widehat{E}^{\prime} \rightarrow \widehat{E}$ defined over $\mathcal{O}_{v}$ with $F_{\sigma}(0)=0$ (Streng proves this for number fields, but the proof works for any local field). It follows immediately that if $v(x(P))<0$, then as $F_{\sigma}(z) \in \mathcal{O}_{v}[[z]]$ vanishes at 0 ,

$$
v\left(B_{\sigma(P)}\right)=v\left(F_{\sigma}(z)\right) \geq v(z)=v\left(B_{P}\right) .
$$

If $E$ is minimal as well, we may apply the same argument to the dual isogeny $\widehat{\sigma}: E \rightarrow E^{\prime}$, noting that the composition is $[m]$. The argument above now tells us that

$$
v\left(B_{\sigma(P)}\right) \leq v\left(B_{m P}\right) \leq v\left(B_{P}\right)+v(m) .
$$

LEMMA 2.4. Let $E$ and $E^{\prime}$ be minimal elliptic curves defined over $K$, and let $\sigma: E^{\prime} \rightarrow E$ be an isogeny of degree $m$. Then

$$
h(E) \ll h\left(E^{\prime}\right)+h(m) .
$$

Proof. In the number field case, this is a consequence of the normalizing lemma of Masser and Wüstholz [20]. Note that Masser and Wüstholz use the quantity

$$
w(E)=\max \left(1, h\left(g_{2}\right), h\left(g_{3}\right)\right)
$$

to measure the "size" of an elliptic curve, where $g_{2}$ and $g_{3}$ are the usual invariants, but $h(E)$ as defined above satisfies $w(E) \ll h(E) \ll w(E)$. The 
argument in [20] produces some isomorphic copy of $E$, say $E^{*} / K$ such that $h\left(E^{*}\right) \ll h\left(E^{\prime}\right)+\log m$. Since $E$ and $E^{*}$ are isomorphic, and $E$ is minimal, we have $h(E) \leq h\left(E^{*}\right)$.

If $K$ is a function field, this property is trivial by the boundedness of the Szpiro ratio, together with the inequality $\operatorname{deg}\left(\Delta_{E^{\prime}}\right) \leq 6 \operatorname{deg}\left(\Delta_{E}\right)$. To see this, note first that $E$ and $E^{\prime}$ have the same conductor, by [13, VII, Corollary 7.2]. The assertion is then a consequence of [23, Theorem 0.1], which bounds the conductor of $E^{\prime}$ in terms of $6 \operatorname{deg}\left(\Delta_{E^{\prime}}\right)$, since the degree of the conductor of $E$ is a lower bound for $\operatorname{deg}\left(\Delta_{E}\right)$. Note that if we assume the $a b c$ conjecture for $K$, we may do the same thing for number fields, but a dependence on $m$ still exists.

Now we return to the main theme of this section, by showing how to reduce the problem to an application of Siegel's theorem.

Definition 2.5. Given $0<\varepsilon<1$, together with a constant $C>0$, say that $P \in E(K)$ is $(\varepsilon, C)$-quasi-integral if

$$
h\left(B_{P}\right) \leq \varepsilon \widehat{h}(P)+C .
$$

Siegel's theorem, in its strong form, is the statement that for any $\varepsilon<1$ and any constant $C$, there are only finitely many $(\varepsilon, C)$-quasi-integral points in $E(K)$. Work of Silverman [28] refines this claim, showing that the number of $(\varepsilon, C)$-quasi-integral points in a subgroup $\Gamma \subseteq E(K)$ may be bounded solely in terms of $\varepsilon, C, \operatorname{rank}(\Gamma)$, and a lower bound on $\varrho(P, E)$ for non-torsion $P \in \Gamma$. Elliptic divisibility sequences are essentially rank one subgroups of $E(K)$, and so under Lang's conjecture a uniform bound is obtained on the number of $(\varepsilon, C)$-quasi-integral points in an elliptic divisibility sequence. Note that if

$$
C h(E)>\delta \widehat{h}(n P)
$$

then $|n| \leq \sqrt{C /(\delta \varrho(P, E))}$.

In particular, if we apply Silverman's version of Siegel's theorem to a rank one subgroup of $E(K)$ generated by some point $P$ with $\varrho(P, E)$ greater than some uniform value, some dependence of $C$ on $E$ is acceptable (in the sense that a uniform quantitative result is recoverable), as long as $C=O(h(E))$.

It will be demonstrated that if $P$ is the image of a $K$-rational point under an isogeny, then $B_{n P} / B_{P}$ being a power of a prime is a non-trivial quasi-integrality condition. The function field case indicates that one would not expect this to be true more generally.

Lemma 2.6. Let $\sigma: E^{\prime} \rightarrow E$ be an isogeny of minimal elliptic curves over $K$, and suppose that $P=\sigma\left(P^{\prime}\right)$ for some $P^{\prime} \in E^{\prime}(K)$. If $B_{n P} / B_{P}$ is a power of a single prime, then either $n P$ is $\left(\varepsilon_{1}, C_{1}\right)$-quasi-integral, with $\varepsilon_{1}=1 / n^{2}+1 / m$ and $C_{1}=O(h(E)+h(m)+h(n))$, or $n P^{\prime}$ is $\left(\varepsilon_{2}, C_{2}\right)$-quasiintegral, with $\varepsilon_{2}=m / n^{2}$ and $C_{2}=O\left(h\left(E^{\prime}\right)+h(m)\right)$. 
Proof. Suppose that $B_{n P} / B_{P}$ is a power of a single prime. Note that, by Lemma 2.3, $v\left(B_{n P^{\prime}}\right) \leq v\left(B_{n P}\right)$ for all $v \in M_{K}^{0}$. Suppose, for the moment, that $v\left(B_{n P^{\prime}}\right) \leq v\left(B_{P}\right)$ for all $v \in M_{K}^{0}$. In this case,

$$
\begin{aligned}
h\left(B_{n P^{\prime}}\right) & \leq h\left(B_{P}\right) \leq h(P) \leq \widehat{h}(P)+O(h(E)) \\
& \leq \frac{m}{n^{2}} \widehat{h}\left(n P^{\prime}\right)+O\left(h\left(E^{\prime}\right)+h(m)\right) .
\end{aligned}
$$

Now suppose that this is not the case, in other words, $v\left(B_{n P^{\prime}}\right)>v\left(B_{P}\right)$ for some prime $v \in M_{K}^{0}$. Then $v\left(B_{n P} / B_{P}\right)>0$, and this is the only prime for which this happens. Furthermore, from Lemma 2.3,

$$
v\left(B_{n P} / B_{P}\right) \leq v\left(B_{n P}\right) \leq v\left(B_{n P^{\prime}}\right)+h(m) .
$$

In particular,

$$
\begin{aligned}
h\left(B_{n P}\right) & \leq h\left(B_{P}\right)+h\left(B_{n P^{\prime}}\right)+h(m)+h(n) \\
& \leq \widehat{h}\left(B_{P}\right)+\widehat{h}\left(B_{n P^{\prime}}\right)+O\left(h(E)+h\left(E^{\prime}\right)+h(m)+h(n)\right) \\
& \leq\left(\frac{1}{n^{2}}+\frac{1}{m}\right) \widehat{h}(n P)+O(h(E)+h(m)+h(n)) .
\end{aligned}
$$

It has been known for a long time that sequences of the form (1) do produce new primes in a weaker sense. Zsigmondy's theorem [35] guarantees a new prime factor for all terms with index $n>6$. The definition is made precise now.

Definition 2.7. A non-zero term $B_{n}$ in a sequence $\left(B_{n}\right)$ of elements in $\mathcal{O}_{K}$ has a primitive divisor $d$ if

(I) $d$ is not a unit,

(II) $d \mid B_{n}$,

(III) $\operatorname{gcd}\left(B_{m}, d\right)$ is a unit for all $m<n$ with $B_{m} \neq 0$.

Silverman [29] proved the elliptic analogue of Zsigmondy's theorem: in any elliptic divisibility sequence $B=\left(B_{n}\right)$, all the terms from some index onwards will have a primitive divisor. This theorem has been proved in a strong uniform manner in [17] (see also [10] and [14]) and will be used crucially in the following proof.

Proof of Theorem 2.1 in the rational case. It will be shown that for every $\delta>0$ there is a constant $M_{\delta}$, depending only on $\delta$, such that for every pair of minimal elliptic curves $E^{\prime}, E / \mathbb{Q}$ equipped with a non-trivial isogeny $\sigma: E^{\prime} \rightarrow E$, and every point $P=\sigma\left(P^{\prime}\right)$ with $\varrho\left(P^{\prime}, E^{\prime}\right) \geq \delta$, there are at most $M_{\delta}$ values $n$ such that $B_{n P} / B_{P}$ is a prime power.

The style of proof depends upon whether the isogeny is cyclic. Firstly, assume that $P \in k E(\mathbb{Q})$ for some integer $k \geq 2$. Then $P \in q E(\mathbb{Q})$ for some prime $q$, and it may be assumed that $k$ is prime. 
Suppose there are distinct non-archimedean valuations $v_{1}$ and $v_{2}$ such that

$$
v_{1}\left(B_{n k P^{\prime}}\right)>0 \quad \text { and } \quad v_{1}\left(B_{k P^{\prime}}\right)=0
$$

and

$$
v_{2}\left(B_{n P^{\prime}}\right)>0 \quad \text { and } \quad v_{2}\left(B_{\operatorname{gcd}(k, n) P^{\prime}}\right)=0 .
$$

Then clearly $v_{1}\left(B_{n P} / B_{P}\right)>0$. On the other hand, $v_{2}\left(B_{k P^{\prime}}\right)=0$, as

$$
v_{2}\left(B_{\operatorname{gcd}(k, n) P^{\prime}}\right)=\min \left(v_{2}\left(B_{n P^{\prime}}\right), v_{2}\left(B_{k P^{\prime}}\right)\right),
$$

and so $v_{2}\left(B_{n P} / B_{P}\right)>0$. Thus if $B_{n P} / B_{P}$ is a prime power, then either

(a) the primes dividing $B_{n k P^{\prime}}$ are at most those dividing $B_{k P^{\prime}}$, or

(b) the primes dividing $B_{n P^{\prime}}$ are at most those dividing $B_{\operatorname{gcd}(k, n) P^{\prime}}$.

Let $Z\left(P^{\prime}, E^{\prime}\right)$ denote the set of $s$ such that $B_{s P^{\prime}}$ has no prime divisors other than those dividing $B_{t P^{\prime}}$ for $t<s$. In case (a), the term $B_{n k P^{\prime}}$ in the elliptic divisibility sequence defined by $P^{\prime}$ has no primitive divisor, and so $n k \in Z\left(P^{\prime}, E^{\prime}\right)$. If (b) holds, and if $\operatorname{gcd}(k, n)<n$, then $n \in Z\left(P^{\prime}, E^{\prime}\right)$. Thus, if $B_{n P} / B_{P}$ is a prime power, then either $n \mid k$ (and recall that $k$ is assumed to be prime) or $n \in Z\left(P^{\prime}, E^{\prime}\right) \cup k^{-1} Z\left(P^{\prime}, E^{\prime}\right)$. By Theorem 7 of $[17]$, \#Z( $\left.P^{\prime}, E^{\prime}\right)$ may be bounded solely in terms of $\varrho\left(P^{\prime}, E^{\prime}\right)$ (note that the statement in [17] is not in terms of $\varrho\left(P^{\prime}, E^{\prime}\right)$, but a simple modification of the proof shows that this is true). Thus the number of $n$ such that $B_{n P} / B_{P}$ is a prime power is bounded by some $M_{\delta, 1}$ which depends only on $\delta$.

So now suppose $P \notin k E(\mathbb{Q})$ for any integer $k \geq 2$. It follows that $\sigma$ is a composition of cyclic isogenies over $\mathbb{Q}$, and so it may be assumed (without loss of generality) that $\sigma$ itself is cyclic. In particular, there are (by work of Mazur [21]) only finitely many possible values for $m=\operatorname{deg}(\sigma)$. Thus Lemma 2.6, upon replacing $h(m)$ with a sufficiently large constant, tells us that if $B_{n P} / B_{P}$ is a prime power, then either $n P$ is $\left(\varepsilon_{1}, C_{1}\right)$-quasi-integral, with $\varepsilon_{1}=1 / n^{2}+1 / m$ and $C_{1}=O(h(E)+h(n))$, or $n P^{\prime}$ is $\left(\varepsilon_{2}, C_{2}\right)$-quasiintegral, with $\varepsilon_{2}=m / n^{2}$ and $C_{2}=O\left(h\left(E^{\prime}\right)\right)$. If $m / n^{2} \leq 3 / 4$, there is a bound on the number of points satisfying the latter condition depending only on $\varrho\left(P^{\prime}, E^{\prime}\right)$. Clearly, the number of $n$ such that $m / n^{2}>3 / 4$ is bounded absolutely.

Now note that $\varrho\left(P^{\prime}, E^{\prime}\right) \geq \delta$ and Lemma 2.4 implies that $\varrho(P, E) \geq \delta_{2}$ for some $\delta_{2}>0$. Thus, the first condition above becomes

$$
h\left(B_{n P}\right) \leq\left(\frac{1}{n^{2}}+\frac{1}{m}\right) \widehat{h}(n P)+O(h(E)+h(n)) .
$$

Finally, for any $C$ and any $\delta_{3}>0$, we can ensure that $C h(n)<\delta_{3} \widehat{h}(n P)$ by taking $n$ large enough. Thus the above becomes

$$
h\left(B_{n P}\right) \leq\left(\frac{1}{n^{2}}+\frac{1}{m}+\delta_{3}\right) \widehat{h}(n P)+O(h(E))
$$


for sufficiently large $n$, where $\delta_{3}$ may be taken as any positive value. Applying the quantitative Siegel theorem completes the proof.

REMARK 2.8. Work of the second author $[15,16]$, in the case $K=\mathbb{Q}$, also assuming Lang's conjecture, can be applied to produce a uniform bound $M$ such that for any elliptic divisibility sequence generated by a magnified point $P$ on a minimal curve, there are at most two values $n>M$ such that $B_{n P}$ is a prime power.

In the function field case, a stronger version of Theorem 2.1 will be proved, namely, that there is a uniform bound $N_{0}$ such that $B_{n} / B_{1}$ is not a prime power for all $n \geq N_{0}$. First, one lemma is required.

Lemma 2.9. Let $K=\mathbb{Q}(t)$ and let $B=\left(B_{n}\right)$ be an elliptic divisibility sequence arising from a non-torsion point on a minimal elliptic curve defined over $K$. There is a uniform bound on the indices $n$ for which $B_{n}$ fails to have a primitive divisor.

Lemma 2.9 will be proved in an explicit way later - see Theorem 3.10.

Proof of Theorem 2.1 in the function field case. Lemma 2.6 shows that if $B_{n P} / B_{P}$ is a prime power, then

$$
h\left(B_{n P}\right) \leq\left(\frac{1}{m}+\frac{1}{n^{2}}\right) \widehat{h}(n P)+O(h(E))
$$

or

$$
h\left(B_{n P^{\prime}}\right) \leq \frac{m}{n^{2}} \widehat{h}\left(n P^{\prime}\right)+O\left(h\left(E^{\prime}\right)\right) .
$$

If $n \geq \sqrt{2 m}$ and $m \geq 2$, then this implies

or

$$
h\left(B_{n P}\right) \leq \frac{3}{4} \widehat{h}(n P)+O(h(E))
$$

$$
h\left(B_{n P^{\prime}}\right) \leq \frac{1}{2} \widehat{h}\left(n P^{\prime}\right)+O\left(h\left(E^{\prime}\right)\right),
$$

respectively. As $\varrho(E, P)$ and $\varrho\left(E^{\prime}, P^{\prime}\right)$ may be bounded below by some absolute, positive value, it follows that the number of such $n$ must be bounded uniformly.

To show that $n$ itself must be bounded, note that if $P \in k E(\mathbb{Q}(t))$ for some integer $k \geq 2$ then the existence of a primitive divisor may be used exactly as in the proof in the rational case - the boundedness of $n$ follows from Lemma 2.9. In the case of a cyclic isogeny, the structure of the proof follows the rational case. The differences are: Lang's conjecture is known to be true; a uniform bound for the degree of the isogeny follows from the existence of the same in the rational case since, for any isogeny over of elliptic curves over $\mathbb{Q}(t)$, some specialization will be an isogeny of elliptic 
curves over $\mathbb{Q}$; and Lemma 2.9 implies a quantitative Siegel theorem in which the index $n$ is bounded.

That $n$ itself may be bounded in the above argument is already suggested by Proposition 8.2 of [12]. In Section 3 below we will work out some explicit bounds, in particular the explicit bound given in Theorem 1.5.

\section{The function field case}

3.1. Explicit bounds for $\mathbb{Q}(t)$. Over $\mathbb{Q}(t)$, Theorem 1.5 raises the possibility of finding all the irreducible terms in particular cases. The bound of 4160366 comes from applying the general version of Lang's conjecture [12], assuming also the maximal possible degree for a cyclic isogeny. As it stands, this is useless: checking the terms coming from smaller indices involves testing rational polynomials for irreducibility which have degrees well in excess of one billion. In particular cases, the problem is circumvented by obtaining a more nuanced version of Lang's conjecture (which takes account of the reduction of small multiples of $P$, see Proposition 3.5) together with Theorem 3.12, which highlights the dependence upon the degree of the isogeny. This technique allows us to exhibit examples of elliptic divisibility sequences where all of the prime (= irreducible) terms can be computed.

Example 3.1. Consider

$$
E: y^{2}=x^{3}+t^{2}\left(1-t^{2}\right) x \quad \text { with } P=\left[t^{2}, t^{2}\right] \text {. }
$$

Then $B_{n} / B_{1}=B_{n}$ fails to be a prime power for all $n \geq 3$. Note that the point $P$ is magnified by a 2 -isogeny as detailed in [2, Chapter 14].

On the other hand, it is worth probing the deeper difference between the cases when $K=\mathbb{Q}$ and $K=\mathbb{Q}(t)$, which is probably at work in general. It is easy to prove that the polynomial $\left(t^{n}-1\right) /(t-1)$ is irreducible for every prime index $n$. An analogous statement seems to be true for some elliptic divisibility sequences over function fields. The two curves in Example 3.2 appeared in [12] as examples where the global canonical height of a point is small:

Example 3.2. The point $P=[0,0]$ on the curve

$$
E: y^{2}+x y+(t+1) t^{2} y=x^{3}+t^{2} x^{2}
$$

produces terms $B_{n P}$ which are irreducible for all primes $n$ from 5 to 199 .

The point $P=[0,0]$ on the curve

$$
E: y^{2}+x y+(t+1) t^{3} y=x^{3}+t^{3} x^{2}
$$

produces terms $B_{n P}$ which are irreducible for all primes $n$ from 5 to 199 .

More surprisingly, there seem to be examples where irreducibility occurs along prime indices in a fixed residue class. 
Example 3.3. The point $P=[1-t, 1-t]$ on the curve

$$
E: y^{2}=x^{3}+t(1-t) x
$$

produces irreducible terms $B_{n P}$ whenever $n \leq 79$ is a prime congruent to 3 $(\bmod 4)$.

That $B_{n P}$ is composite for any prime $n \equiv 1(\bmod 4)$ is not surprising, in light of results of Streng [33] for elliptic divisibility sequences arising from elliptic curves (over number fields) with complex multiplication.

3.2. Lang's conjecture. We do not propose to make explicit the argument given in Section 2. Instead, the technique is to work with local heights and explicit bounds for the difference between the naïve and canonical heights. When $K=\mathbb{Q}(t)$, the infinite place $\infty$ corresponds to the valuation - deg. Without loss of generality, assume that $E$ is given by a short Weierstrass form

$$
y^{2}=x^{3}+A x+B
$$

with $A, B \in \mathbb{Q}[t]$, so that $\Delta_{E}=-16\left(4 A^{3}+27 B^{2}\right)$. Then the $j$-invariant $j_{E}$ of $E$ is given by

$$
j_{E}=\frac{-1728(4 A)^{3}}{\Delta_{E}}
$$

and the height $h(E)$ of the curve $E$ is

$$
h(E)=\frac{1}{12} \max \left(h\left(j_{E}\right), h\left(\Delta_{E}\right)\right)=\frac{1}{12} \max (3 \operatorname{deg}(A), 2 \operatorname{deg}(B)) .
$$

For $v$ any place of $K$, denote by $\lambda_{v}(P)$ the Néron local height of $P$ at $v$, and by $h_{v}(P)$ the naïve local height

$$
h_{v}(P)=\frac{1}{2} \max (0,-v(x(P))) .
$$

The first lemma compares the heights of the $j$-invariant and discriminant of $E$.

Lemma 3.4. With $j_{E}$ as in (3),

$$
h\left(j_{E}\right) \leq \frac{3}{2} \operatorname{deg}\left(\Delta_{E}\right) .
$$

Proof. Let $L$ be the smallest Galois extension of $\mathbb{Q}(t)$ containing the roots $\delta_{1}, \delta_{2}$ and $\delta_{3}=-\delta_{1}-\delta_{2}$ of $x^{3}+A x+B$. Let $v$ be a place of $L$ above the place at infinity. Since $x^{3}+A x+B$ is monic with polynomial coefficients, $v_{\mathcal{P}}\left(\delta_{i}\right) \geq 0$ for every finite place $\mathcal{P}$ of $L$, so that $v_{\mathcal{P}}\left(\delta_{i}-\delta_{j}\right) \geq 0$. Summing over all finite places of $L$, and using the fact that $\delta_{i}-\delta_{j}$ has the same valuation at all places of the Galois extension $L$ above the infinite place, we deduce that $v\left(\delta_{i}-\delta_{j}\right) \leq 0$. 
Since $v$ is non-archimedean, at most one of the valuations $v\left(\delta_{1}-\delta_{2}\right)$, $v\left(2 \delta_{1}+\delta_{2}\right)$ and $v\left(\delta_{1}+2 \delta_{2}\right)$ can be different from $\min \left(v\left(\delta_{1}\right), v\left(\delta_{2}\right)\right)$. In particular, since $\Delta_{E}=\left(\left(\delta_{1}-\delta_{2}\right)\left(2 \delta_{1}+\delta_{2}\right)\left(\delta_{1}+2 \delta_{2}\right)\right)^{2}$, it follows that $v\left(\Delta_{E}\right) \leq$ $4 \min \left(v\left(\delta_{1}\right), v\left(\delta_{2}\right)\right)$. Hence

$$
v\left(B^{2}\right)=2\left(v\left(\delta_{1}\right)+v\left(\delta_{2}\right)+v\left(\delta_{1}+\delta_{2}\right)\right) \geq 6 \min \left(v\left(\delta_{1}\right), v\left(\delta_{2}\right)\right) \geq \frac{3}{2} v\left(\Delta_{E}\right) .
$$

Summing over all places $v$ of $L$ above the place at infinity gives

$$
\operatorname{deg}\left(B^{2}\right) \leq \frac{3}{2} \operatorname{deg}\left(\Delta_{E}\right)
$$

The lemma is now a consequence of the inequality

$$
\operatorname{deg}\left(A^{3}\right) \leq \max \left(\operatorname{deg}\left(B^{2}\right), \operatorname{deg}\left(\Delta_{E}\right)\right) \leq \frac{3}{2} \operatorname{deg}\left(\Delta_{E}\right)
$$

together with the definition $j_{E}=-1728(4 A)^{3} / \Delta_{E}$ of the $j$-invariant of $E$.

The following is a version of Lang's conjecture which was proved in the function field case by Hindry and Silverman [12]. The explicit constants obtained here, in certain special cases, use the same basic methods.

Proposition 3.5. There is a constant $c>0$ such that, for all nontorsion points $P \in E(K)$,

$$
c \operatorname{deg}\left(\Delta_{E}\right) \leq \widehat{h}(P),
$$

where $c$ can be taken to be the following:

- in the general case, $c=10^{-9.2}$;

- if $P$ has everywhere good reduction, $c=\frac{1}{12}$;

- if $P$ has everywhere good reduction except at infinity, $c=\frac{1}{16}$;

- if $E$ does not have split multiplicative reduction (in particular, if $E$ is isotrivial), $c=\frac{1}{1728}$;

- if $E$ does not have split multiplicative reduction except at infinity (in particular, if $E$ has a polynomial $j$-invariant), $c=\frac{1}{2304}$.

Proof. The first bound is the second remark on Theorem 6.1 in [12]. Assume now that $P$ has everywhere good reduction except maybe at infinity. Let $v$ be a valuation different from the valuation at infinity. The coefficients of the chosen Weierstrass equation are polynomials. Following [18, III, Theorem 4.5], the Néron local height $\lambda_{v}$ at $v$ satisfies

$$
\lambda_{v}(P)=\frac{1}{2} \max (-v(x(P)), 0)+\frac{1}{12} v\left(\Delta_{E}\right) \geq \frac{1}{12} v\left(\Delta_{E}\right),
$$

while the Néron local height $\lambda_{\infty}$ at infinity satisfies

$$
\lambda_{\infty}(P) \geq \frac{1}{2} \max (0, \operatorname{deg}(X(P)))+\frac{1}{24} \min \left(0,-h\left(j_{E}\right)\right) \geq-\frac{1}{24} h\left(j_{E}\right)
$$


for some function $X$ on $E(K)$. Summing over all places gives

$$
\widehat{h}(P) \geq \frac{1}{12} \operatorname{deg}\left(\Delta_{E}\right)-\frac{1}{24} h\left(j_{E}\right) .
$$

In particular, Lemma 3.4 implies

$$
\widehat{h}(P) \geq\left(1-\frac{1}{4}\right) \frac{1}{12} \operatorname{deg}\left(\Delta_{E}\right)=\frac{1}{16} \operatorname{deg}\left(\Delta_{E}\right)
$$

and the third bound follows.

Now assume $P$ also has good reduction at infinity. Since the coefficients in the Weierstrass equation are not integral at infinity, a transformation is needed to an $\infty$-minimal Weierstrass equation, with discriminant $\Delta_{\infty}$. Since the Néron local height does not depend on the choice of Weierstrass equation,

$$
\lambda_{\infty}(P) \geq \frac{1}{12} v_{\infty}\left(\Delta_{\infty}\right) \geq 0 .
$$

Summing over all places as before, we get

$$
\widehat{h}(P) \geq \frac{1}{12} \sum_{v \neq \infty} v\left(\Delta_{E}\right)=-\frac{1}{12} v_{\infty}\left(\Delta_{E}\right)=\frac{1}{12} \operatorname{deg}\left(\Delta_{E}\right),
$$

which gives the second bound.

When $E$ does not have split multiplicative reduction at $v$, the KodairaNéron theorem asserts that $12 P$ has good reduction at $v$. Hence the bounds for $\widehat{h}(P)$ and the functoriality of the canonical height $\widehat{h}(12 P)=144 \widehat{h}(P)$ give the final two bounds.

REMARK 3.6. In special cases, one can improve the constants in Proposition 3.5 by a more careful analysis of the reduction types at bad primes. Moreover, even when $E$ has split multiplicative reduction, the KodairaNéron theorem gives a good constant also when there is a small common multiple of all the valuations of the discriminant $\Delta_{E}$ (for example, if it is square-free).

3.3. Naïve and canonical heights. In order to estimate the difference between the naïve and canonical heights of a point, pass to the local heights. The most difficult of these is the height at infinity, which is addressed first.

Lemma 3.7. For $P \in E(K)$, $-\frac{5}{6}-h(E)+\frac{1}{24} \min \left(0, v_{\infty}(j)\right) \leq \lambda_{\infty}(P)-h_{\infty}(P) \leq h(E)+\frac{5}{6}-\frac{1}{12} \operatorname{deg}\left(\Delta_{E}\right)$.

Proof. The coefficients $A$ and $B$ of the minimal Weierstrass equation (2) are not $v_{\infty}$-integral. A Weierstrass equation of $E$ with $v_{\infty}$-integral coefficients is given by

$$
Y^{2}=X^{3}+t^{-4 r} A X+B t^{-6 r},
$$


where $r$ is the smallest integer greater than or equal to $h(E)$. The corresponding change of variables is given by $x=t^{2 r} X$ and $y=t^{3 r} Y$ and the discriminant of this equation is $\Delta_{\infty}=t^{-12 r} \Delta_{E}$.

By [18, III, Theorem 4.5], the Néron local height $\lambda_{\infty}$ satisfies

$$
\frac{1}{24} \min \left(0, v_{\infty}(j)\right) \leq \lambda_{\infty}(P)-\frac{1}{2} \max \left(0,-v_{\infty}(X(P))\right) \leq \frac{1}{12} v_{\infty}\left(\Delta_{\infty}\right) .
$$

Since $2 \lambda_{\infty}(P)-r \leq \frac{1}{2} \max (0,-2 r+\operatorname{deg}(x(P))) \leq \lambda_{\infty}(P)$, this can be reformulated in terms of $x(P), \Delta_{E}$ and $r$ as

$$
-r+\frac{1}{24} \min \left(0, v_{\infty}(j)\right) \leq \lambda_{\infty}(P)-h_{\infty}(P) \leq r-\frac{1}{12} \operatorname{deg}\left(\Delta_{E}\right) .
$$

The number $r$ is the smallest integer greater than or equal to $h(E)$, which is at most $h(E)+\frac{5}{6}$, and the result follows.

Now the difference between naïve and canonical heights can be bounded.

Proposition 3.8. For $P \in E(K)$,

$$
-\frac{3}{16} \operatorname{deg}\left(\Delta_{E}\right)-\frac{5}{6} \leq \widehat{h}(P)-h(P) \leq \frac{1}{8} \operatorname{deg}\left(\Delta_{E}\right)+\frac{5}{6} .
$$

Proof. By [18, III, Theorem 4.5], the Néron local height $\lambda_{v}$ at any finite place $v$ satisfies

$$
\frac{1}{24} \min (0, v(j)) \leq \lambda_{v}(P)-h_{v}(P) \leq \frac{1}{12} v\left(\Delta_{E}\right) .
$$

Together with Lemma 3.7, and summing over all places, this gives

$$
-\frac{5}{6}-h(E)-\frac{1}{24} h(j) \leq \widehat{h}(P)-h(P) \leq h(E)+\frac{5}{6} .
$$

To conclude we notice that $h(j) \leq 12 h(E)$ and use the fact that $h(E) \leq$ $\frac{1}{8} \operatorname{deg}\left(\Delta_{E}\right)$ from Lemma 3.4 .

This subsection concludes with an estimate on the degree of the denominator of $x(P)$, in terms of the (naïve) height of $P$. This, in particular the fact that the constant $\frac{3}{4}$ is large enough, is crucial to the explicit bounds later.

Lemma 3.9. For $P \in E(K)$,

$$
\frac{3}{4} h(P)-\frac{3}{16} \operatorname{deg}\left(\Delta_{E}\right) \leq \operatorname{deg}\left(B_{P}\right) .
$$

Proof. This uses a standard approach to Siegel's theorem. Assume that $h(P)=\frac{1}{2} \operatorname{deg}\left(A_{P}\right)$ (or we are done). Denote by $K_{\infty}$ the completion of $K$ at the infinite valuation, and by $L$ the algebraic closure of $K_{\infty}$. Let $v$ be the valuation on $L$ which extends the valuation $-\operatorname{deg}$ on $K_{\infty}$. Inserting the coordinates of $P$ into the equation for $E$ gives

$$
C_{P}^{2}=\left(A_{P}-\delta_{1} B_{P}^{2}\right)\left(A_{P}-\delta_{2} B_{P}^{2}\right)\left(A_{P}-\delta_{3} B_{P}^{2}\right) .
$$


This may be factored in $L$ as

$$
C_{i}^{2}=A_{P}-\delta_{i} B_{P}^{2}
$$

because the factors of $\Delta_{E}$ are squares in $L$ (so they may be absorbed into the $\left.C_{i}, i=1,2,3\right)$.

As in the proof of Lemma 3.4, $v\left(\Delta_{E}\right) \leq \frac{1}{4} v\left(\delta_{i}\right)$ for each $i$. If $v\left(A_{P}\right) \geq$ $v\left(\delta_{i} B_{P}^{2}\right)$ then we have $v\left(A_{P}\right) \geq 2 v\left(B_{P}\right)+\frac{1}{4} v\left(\Delta_{E}\right)$ and nothing further is needed. So consider the case when $v\left(A_{P}\right)<v\left(\delta_{i} B_{P}^{2}\right)$ for $i=1,2,3$, or more precisely the case when

$$
v\left(A_{P}\right)=2 v\left(C_{i}\right), \quad i=1,2,3 .
$$

For $i \neq j$,

$$
\left(\delta_{j}-\delta_{i}\right) B_{P}^{2}=C_{i}^{2}-C_{j}^{2}=\left(C_{i}+C_{j}\right)\left(C_{i}-C_{j}\right)
$$

therefore

$$
B_{i j+}^{2}=C_{i}+C_{j} \quad \text { and } \quad B_{i j-}^{2}=C_{i}-C_{j} .
$$

Without loss of generality, assume $v\left(B_{i j+}\right) \leq v\left(B_{i j-}\right)$.

We assert that (at least) one of the following two inequalities holds:

$$
\begin{array}{ll}
v\left(B_{12+}\right) & \geq \frac{2}{3} v\left(B_{P}\right)+\frac{1}{2} v\left(\delta_{2}-\delta_{1}\right) \quad \text { or } \\
v\left(B_{13+}\right) & \geq \frac{2}{3} v\left(B_{P}\right)+\frac{1}{2} v\left(\delta_{3}-\delta_{1}\right) .
\end{array}
$$

To prove this assume that

$$
\begin{aligned}
& v\left(B_{12+}\right)<\frac{2}{3} v\left(B_{P}\right)+\frac{1}{2} v\left(\delta_{2}-\delta_{1}\right) \quad \text { and } \\
& v\left(B_{13+}\right)<\frac{2}{3} v\left(B_{P}\right)+\frac{1}{2} v\left(\delta_{3}-\delta_{1}\right) .
\end{aligned}
$$

This forces

$$
v\left(B_{12-}\right)>\frac{1}{3} v\left(B_{P}\right) \quad \text { and } \quad v\left(B_{13-}\right)>\frac{1}{3} v\left(B_{P}\right) .
$$

Then Siegel's relation $\left(C_{1}-C_{2}\right)+\left(C_{2}-C_{3}\right)=C_{1}-C_{3}$ forces

$$
v\left(B_{23-}\right)>\frac{1}{3} v\left(B_{P}\right) \text {. }
$$

Since $\operatorname{lcm}\left(B_{12+}^{2}, B_{13+}^{2}\right)$ divides $B_{P}^{2}\left(\delta_{2}-\delta_{1}\right)\left(\delta_{3}-\delta_{1}\right)$, the inequalities (5) imply $v\left(\operatorname{gcd}\left(B_{12+}, B_{13+}\right)\right)=v\left(B_{12+}\right)+v\left(B_{13+}\right)-v\left(\operatorname{lcm}\left(B_{12+}, B_{13+}\right)\right)<\frac{1}{3} v\left(B_{P}\right)$. Now Siegel's relation $\left(C_{1}+C_{2}\right)-\left(C_{1}+C_{3}\right)=C_{2}-C_{3}$ shows that $\operatorname{gcd}\left(B_{12+}, B_{13+}\right)$ divides $B_{23-}$. In particular,

$$
\frac{1}{3} v\left(B_{P}\right)<v\left(B_{23-}\right) \leq \operatorname{gcd}\left(B_{12+}, B_{13+}\right)<\frac{1}{3} v\left(B_{P}\right),
$$

which is absurd. 
Hence one of the inequalities (4) holds, say the first. Since

$$
v\left(B_{12-}\right) \geq v\left(B_{12+}\right) \quad \text { and } \quad 2 C_{1}=B_{12+}^{2}+B_{12-}^{2},
$$

it follows that

$$
\frac{1}{2} v\left(A_{P}\right)=v\left(C_{1}\right) \geq \frac{4}{3} v\left(B_{P}\right)+v\left(\delta_{2}-\delta_{1}\right) \geq \frac{4}{3} v\left(B_{P}\right)+\frac{1}{4} v\left(\Delta_{E}\right) .
$$

To conclude, recall that $v$ extends - deg.

\subsection{Primitive divisors}

TheOREM 3.10. Let $P \in E(K)$ be a non-torsion point and let $B=$ $\left(B_{n}\right)$ be the elliptic divisibility sequence arising from $P$. There is a uniform constant $N_{0}$ such that for all $n \geq N_{0}, B_{n}$ has a primitive divisor.

- In general, $N_{0}=190000$.

- If $P$ has everywhere good reduction, $N_{0}=7$.

- If $P$ has everywhere good reduction except at infinity, $N_{0}=11$.

- If $E$ is isotrivial, $N_{0}=133$.

- If $E$ has a polynomial $j$-invariant, $N_{0}=151$.

Proof. Suppose the term $B_{n}$ does not have a primitive divisor. Given any irreducible factor $f$, there is a $d<n$ with $f \mid B_{d}$. Since $f$ also divides $B_{\operatorname{gcd}(n, d)}$ we may assume $d$ is actually a divisor of $n$. What is more, the valuation of $B_{n}$ and $B_{d}$ must be the same. It follows that if $B_{n}$ does not have a primitive divisor then $B_{n}$ divides $\prod_{p \mid n} B_{n / p}$; in particular,

$$
\operatorname{deg}\left(B_{n}\right) \leq \sum_{p \mid n} \operatorname{deg}\left(B_{n / p}\right)
$$

Now apply Lemma 3.9 to obtain

$$
\frac{3}{4} h(n P)-\frac{3}{16} \operatorname{deg}\left(\Delta_{E}\right) \leq \sum_{p \mid n} h\left(\frac{n}{p} P\right) .
$$

Proposition 3.8, with the functoriality of the canonical height, gives

$$
\begin{aligned}
\frac{3}{4}\left(h n^{2}-\frac{1}{8} \operatorname{deg}\left(\Delta_{E}\right)\right. & \left.-\frac{5}{6}\right)-\frac{3}{16} \operatorname{deg}\left(\Delta_{E}\right) \\
& \leq h n^{2}\left(\sum_{p \mid n} \frac{1}{p^{2}}\right)+\frac{3}{16} \omega(n) \operatorname{deg}\left(\Delta_{E}\right)+\frac{5}{6} \omega(n),
\end{aligned}
$$

where $\omega(n)$ denotes the number of distinct prime factors of $n$. Re-arranging, dividing by $\operatorname{deg}\left(\Delta_{E}\right) \geq 1$, and applying Lang's conjecture (Proposition 3.5, with $c$ equal to the constant from there) gives

$$
c n^{2}\left(\frac{3}{4}-\sum_{p \mid n} \frac{1}{p^{2}}\right) \leq \frac{87+98 \omega(n)}{96} .
$$


Using

$$
\frac{3}{4}-\sum_{p \mid n} \frac{1}{p^{2}} \geq 0.297 \quad \text { and } \quad \omega(n) \leq \log n / \log 2
$$

firstly bounds $n$ roughly. Then a more careful analysis of small values of $n$ yields the bounds in the theorem.

3.5. Prime powers. Suppose now that $E^{\prime}$ is another elliptic curve over $K$, defined relative to a minimal short Weierstrass equation with discriminant $\Delta_{E^{\prime}}$, and that $\sigma: E^{\prime} \rightarrow E$ is an isogeny of degree $m>1$. The following is a direct consequence of Lemma 2.3.

Lemma 3.11. Suppose $P^{\prime} \in E^{\prime}(K)$ and $P=\sigma\left(P^{\prime}\right)$. Then

$$
B_{P}=B_{P^{\prime}} \widetilde{B}_{P}
$$

with $\widetilde{B}_{P}$ coprime to $B_{P^{\prime}}$.

We already know how to deal with isogenies of the form $[k]$ for some integer $k$, using Theorem 3.10. From now on, we work only with cyclic isogenies. Note that the degree of such an isogeny is bounded as in the rational case (by specialization), $m \leq 163$, by Mazur's famous result [21] (see also [26, p. 265]). The explicit dependence upon the degree is shown in what follows.

TheOREM 3.12. Suppose $B=\left(B_{n P}\right)$ is an elliptic divisibility sequence generated by a rational point $P$ on a minimal elliptic curve. Assume $P$ is magnified by a cyclic isogeny of degree $m$. There are uniform constants $N_{0}$ and $N_{1}$ such that for all

$$
n \geq \max \left(N_{0}, N_{1} \sqrt{m}\right),
$$

$B_{n} / B_{1}$ has at least two distinct prime factors.

- In general, $N_{0}=133034, N_{1}=325865$.

- If $P^{\prime}$ has everywhere good reduction, $N_{0}=12, N_{1}=5$.

- If $P^{\prime}$ has everywhere good reduction except at infinity, $N_{0}=14$, $N_{1}=6$.

- If $E^{\prime}$ is isotrivial, $N_{0}=140, N_{1}=58$.

- If $E^{\prime}$ has a polynomial $j$-invariant, $N_{0}=161, N_{1}=67$.

Proof of Theorem 1.5. The proof follows from Theorem 3.12 for the cyclic case and Theorem 3.10 if the isogeny is $[k]$ for some integer $k$. The worst possible bound comes from assuming we have a cyclic isogeny of degree 163, together with no other special assumptions. 
Proof of Theorem 3.12. Write $B^{\prime}=\left(B_{n}^{\prime}\right)$ for the elliptic divisibility sequence arising from $P^{\prime}$. By Lemma 3.11,

$$
\frac{B_{n}}{B_{1}} B_{1}=B_{n}^{\prime} \widetilde{B}_{n}
$$

If $\operatorname{deg}\left(B_{n} / B_{1}\right)>\operatorname{deg}\left(B_{n}^{\prime}\right)>\operatorname{deg}\left(B_{1}\right)$ then $B_{n} / B_{1}$ has two distinct prime factors. Begin with the first inequality. To bound $\operatorname{deg}\left(B_{n} / B_{1}\right)$ below use Lemma 3.9 and Proposition 3.8. This yields

$$
\operatorname{deg}\left(B_{n}\right)-\operatorname{deg}\left(B_{1}\right)>h\left(\frac{3 n^{2}}{4}-1\right)-\frac{15}{32} \operatorname{deg}\left(\Delta_{E}\right)-\frac{35}{24} .
$$

To bound $\operatorname{deg}\left(B_{n}^{\prime}\right)$ above use the same tools. Note that the functoriality of the canonical height ensures $h=m h^{\prime}$ with $m \geq 2$. Then

(8) $\operatorname{deg}\left(B_{n}^{\prime}\right)<h^{\prime} n^{2}+\frac{3}{16} \operatorname{deg}\left(\Delta_{E^{\prime}}\right)+\frac{5}{6}<\frac{h}{m} n^{2}+\frac{3}{96} \operatorname{deg}\left(\Delta_{E}\right)+\frac{5}{6}$.

The right hand side of (8) is guaranteed to be smaller than the right hand side of $(7)$ if

$$
h\left(\frac{n^{2}}{m}-1\right)>\frac{1}{2} \operatorname{deg}\left(\Delta_{E}\right)+\frac{55}{24} .
$$

Applying Lang's conjecture (Proposition 3.5, with $c$ equal to the constant from there), $n$ is guaranteed to be large enough if

$$
n^{2} \geq 4\left(1+\frac{67}{24 c}\right)
$$

This shows that $\operatorname{deg}\left(B_{n}\right)>\operatorname{deg}\left(B_{n}^{\prime}\right)$ for all $n \geq N_{0}$, say. Substituting in the values for $c$ from Proposition 3.5 gives the stated values for $N_{0}$.

For the second inequality, again use Lemma 3.9 and Proposition 3.8. These give a lower bound for $\operatorname{deg}\left(B_{n}^{\prime}\right)$ of the form

$$
\frac{3}{4} h^{\prime} n^{2}-\frac{9}{32} \operatorname{deg}\left(\Delta_{E}\right)-\frac{15}{24} \text {. }
$$

Similarly, they give rise to an upper bound for $\operatorname{deg}\left(B_{1}\right)$ of the form

$$
\operatorname{deg}\left(B_{1}\right)<h+\frac{3}{16} \operatorname{deg}\left(\Delta_{E}\right)+\frac{5}{6} .
$$

In view of the relation $h=m h^{\prime}$, what we require is guaranteed if

$$
h\left(\frac{3 n^{2}}{4 m}-1\right)>\frac{15}{32} \operatorname{deg}\left(\Delta_{E}\right)+\frac{35}{24} .
$$

Using Lang's conjecture means we can guarantee $\operatorname{deg}\left(B_{n}^{\prime}\right)>\operatorname{deg}\left(B_{1}\right)$ if

$$
n^{2}>m\left(1+\frac{185}{96 c}\right) \text {. }
$$

Inserting the various possibilities for $c$ gives $N_{1}$. 
3.6. Explicit computations. Given any particular example, it is possible to compute $h, \operatorname{deg}\left(\Delta_{E}\right)$ and $m$. These data can be fed into the bounds (9) and (10). Example 3.1 does not satisfy any of the special criteria in Theorem 3.12 so it is best handled this way. The point $P=\left[t^{2}, t^{2}\right]$ on $E$ : $y^{2}=x^{3}+t^{2}\left(1-t^{2}\right) x$ has global canonical height equal to $\frac{1}{2}$. It has bad reduction at $t$ and is the image of a $K$-rational point under a 2 -isogeny. This data is inserted into (9) and (10) to obtain a reasonable bound for the indices $n$ beyond which $B_{n}$ is reducible. Then the smaller indices can easily be checked.

On the other hand, the point $2 P=\left[t^{4}-t^{2}+\frac{1}{4},-t^{6}+\frac{3}{2} t^{4}-\frac{1}{4} t^{2}-\frac{1}{8}\right]$ has good reduction away from infinity, so Theorem 3.12 can be applied directly to obtain a reasonable bound. Then the smaller indices can be checked manually to verify that, for all $n \geq 2$, the terms $B_{n} / B_{1}=B_{n}$ have at least two distinct irreducible factors.

4. The rational case. Explicit results, when the base field is $\mathbb{Q}$, are harder to obtain. This is partly because Lang's conjecture is not known in general. The table in Section 4.2 (see also Silverman [27, Exercise 6.10(d)]) gives examples of rational points with very small ratio $\widehat{h}(P) / \log \Delta$, forcing the constant in Lang's conjecture to be small. This small constant is expected to play a big role in the determination of an explicit upper bound in Theorem 1.4. This makes a reasonable global uniform bound for Theorem 1.4 seem a very distant prospect. On the other hand, it is possible to use our techniques to get reasonable bounds in particular cases, for example, the congruent number curves:

Example 4.1. See [9] for details. For

$$
E: y^{2}=x^{3}-N^{2} x
$$

with $5 \leq N \in \mathbb{N}$ square-free, assume $P$ lies in the image of a 2-isogeny and has $x(P)<0$; then each term $B_{n} / B_{1}$ fails to be a prime power for all $n \geq 8$. If $P$ is integral then $B_{n}$ fails to be a prime power for all $n \geq 3$. The conditions stated seem to be satisfied for quite a few examples with small $N$. For example, when $N=5$ and $P=[-4,6]$ (because $-4+5$ is a square). Similarly, when $N=6$ and $P=[-2,8]$. Frustratingly, each curve also gives rise to elliptic divisibility sequences which are untouchable by our methods; even to the extent that we cannot prove that only finitely many terms are prime.

4.1. The uniform primality conjecture. The heuristic argument in [6] predicts a bound for the number of prime terms in an elliptic divisibility sequence. That argument can be sharpened by making an assumption coming from diophantine analysis to predict a uniform bound, provided the curve is minimal. 
The prime number theorem suggests that the probability that a large integer $N$ is prime is roughly $1 / \log N$. Therefore, if $\left(x_{n}\right)_{n \geq 1}$ is an increasing sequence of positive integers, this suggests that (in the absence of an obvious reason to believe otherwise), the expected number of prime terms $x_{n}$ with $n \leq X$ is approximately

$$
\sum_{n \leq X} \frac{1}{\log x_{n}}
$$

In particular, one should suspect that $x_{n}$ is prime infinitely often if and only if the sum in (11) diverges as $X \rightarrow \infty$. If $x_{n}=\left(a^{n}-b^{n}\right) /(a-b)$, then

$$
\log x_{n} \sim n h(a / b) \quad \text { as } n \rightarrow \infty,
$$

and so the sum diverges like a constant multiple of $\log X$. All the available evidence supports the belief that a sequence of this form with $a$ and $b$ not both $k$ th powers of integers, for some $k>1$, will produce prime terms at that rate. If $x_{n}=B_{n} / B_{1}$, on the other hand,

$$
\log x_{n} \sim n^{2} \widehat{h}(P) \quad \text { as } n \rightarrow \infty,
$$

where $\widehat{h}(P)$ is the global canonical height of $P$. As a consequence, the sum in (11) converges, and it seems likely that $B_{n} / B_{1}$ is prime only finitely often.

A sharpened version of this heuristic argument can be given. Using David's theorem [5] from elliptic transcendence theory, it can be shown that a lower bound for $\log B_{n}$ of the following kind holds:

$$
\log B_{n}>(1-\varepsilon) \widehat{h}(P) n^{2}-C \log n(\log \log n)^{4}
$$

for any $\varepsilon>0$. If one could show, for $E$ minimal, that $C=O(h(E))$, then Lang's conjecture would imply that the sum in (11) is uniformly bounded above. In other words, for elliptic divisibility sequences coming from curves in minimal form, one would expect that the number of prime terms in the sequence $B_{n} / B_{1}$ would be uniformly bounded. Although David's theorem is very strong, currently the best form of the constant $C$ has a polynomial but non-linear dependence upon $h(E)$, as well as a dependence on $\widehat{h}(P)$.

On the other hand, it was suggested to us that the Bateman-Horn conjecture [1] in a suitable form provides a way to contradict the uniform primality conjecture in certain cases. This method requires examples of elliptic divisibility sequences over $\mathbb{Q}(T)$ with infinitely many irreducible terms $B_{n}$. That such sequences exist is suggested by the examples in Section 3.1 but not proven. By specializing, one can expect arbitrarily many prime terms in certain elliptic divisibility sequences over $\mathbb{Q}$. The key question lies with the possibility of specializing in such a way as to produce minimal curves. No answer to that question is known. 
In summary, it is unclear whether or not one should expect the number of prime terms in the sequence $B_{n} / B_{1}$, even for minimal elliptic curves, to be uniformly bounded.

4.2. Explicit computations. In the table below are presented prime terms in sequences generated by the rational points with smallest known height, in 18 cases drawn from Elkies' tables. The computations were performed using PARI-GP [22] (for the first 6 cases) and MAGMA [19] (for the other 12). In the table,

- $E$ is an elliptic curve which is given by a minimal equation as a vector $\left[a_{1}, \ldots, a_{6}\right]$ in Tate's notation;

- $P$ denotes a non-torsion point in $E(\mathbb{Q})$;

- $\left(B_{n}\right)_{n \in \mathbb{N}}$ denotes the elliptic divisibility sequence associated to $P$;

- $h_{0}$ denotes 1000 times the global canonical height of $P$;

- $c_{0}$ denotes 10000 times the ratio $\widehat{h}(P) / h(E)$;

- $N_{2}$ denotes the maximal index for which the primality of $B_{n}$ has been tested;

- $N_{0}$ denotes the number of indices $n \leq N_{2}$ such that $B_{n}=1$;

- $N_{1}$ denotes the number of indices $n \leq N_{2}$ such that $B_{n}$ is a prime number;

- $N_{3}$ is the greatest index $n \leq N_{2}$ such that $B_{n}$ is a prime number.

\begin{tabular}{llcccccr}
\hline \multicolumn{1}{c}{ Minimal model } & \multicolumn{1}{c}{ Point $P$} & $h_{0}$ & $c_{0}$ & $N_{0}$ & $N_{1}$ & $N_{2}$ & $N_{3}$ \\
\hline$[1,1,1,-125615,61201397]$ & {$[7107,594946]$} & 4.45 & 1.06 & 15 & 32 & 4500 & 3719 \\
{$[1,0,0,-141875,18393057]$} & {$[-386,-3767]$} & 4.51 & 1.18 & 15 & 32 & 4900 & 1811 \\
{$[1,-1,1,-3057,133281]$} & {$[591,-14596]$} & 4.86 & 1.65 & 13 & 29 & 4700 & 541 \\
{$[1,1,1,-2990,71147]$} & {$[27,-119]$} & 4.98 & 1.84 & 14 & 32 & 4500 & 829 \\
{$[0,0,0,-412,3316]$} & {$[-18,-70]$} & 5.63 & 2.90 & 11 & 28 & 4300 & 317 \\
{$[1,0,0,-4923717,4228856001]$} & {$[1656,-25671]$} & 5.71 & 1.24 & 14 & 29 & 4300 & 419 \\
{$[1,0,0,-13465,839225]$} & {$[80,485]$} & 5.77 & 17.2 & 15 & 25 & 3000 & 571 \\
{$[1,0,0,-21736,875072]$} & {$[-154,-682]$} & 5.92 & 17.1 & 12 & 25 & 3000 & 953 \\
{$[1,-1,1,-1517,26709]$} & {$[167,-2184]$} & 6.03 & 25.7 & 13 & 22 & 3000 & 1283 \\
{$[1,0,0,-8755,350177]$} & {$[14,473]$} & 6.12 & 18.9 & 15 & 32 & 3000 & 401 \\
{$[1,-1,1,-180,1047]$} & {$[-1,35]$} & 6.42 & 37.1 & 12 & 27 & 3000 & 383 \\
{$[1,0,0,-59852395,185731807025]$} & {$[12680,1204265]$} & 6.56 & 12.0 & 13 & 21 & 3000 & 359 \\
{$[1,0,0,-10280,409152]$} & {$[304,-5192]$} & 6.62 & 20.2 & 13 & 32 & 3000 & 311 \\
{$[0,1,1,-310,3364]$} & {$[-19,52]$} & 6.70 & 36.7 & 12 & 20 & 3000 & 103 \\
{$[1,0,0,-42145813,105399339617]$} & {$[31442,5449079]$} & 6.78 & 14.8 & 14 & 21 & 3000 & 349 \\
{$[1,0,0,-25757,320049]$} & {$[-116,-1265]$} & 6.82 & 19.4 & 14 & 22 & 3000 & 83 \\
{$[1,0,0,-350636,80632464]$} & {$[352,748]$} & 6.91 & 16.6 & 13 & 22 & 3000 & 137 \\
{$[1,0,0,-23611588,39078347792]$} & {$[-3718,-272866]$} & 7.41 & 16.6 & 13 & 23 & 3000 & 109 \\
\hline
\end{tabular}


EXAMPLE 4.2. The largest prime obtained comes from the first pair: the point

$$
P=[7107,594946]
$$

on the curve

$$
E: y^{2}+x y+y=x^{3}+x^{2}-125615 x+61201397
$$

yields a prime term $B_{3719}$ with 26774 decimal digits.

\section{References}

[1] P. T. Bateman and R. A. Horn, A heuristic asymptotic formula concerning the distribution of prime numbers, Math. Comp. 16 (1962), 363-367.

[2] J. W. S. Cassels, Lectures on Elliptic Curves, London Math. Soc. Student Texts 24, Cambridge Univ. Press, Cambridge, 1991.

[3] D. V. Chudnovsky and G. V. Chudnovsky, Sequences of numbers generated by addition in formal groups and new primality and factorization tests, Adv. in Appl. Math. 7 (1986), 385-434.

[4] G. Cornelissen and K. Zahidi, Elliptic divisibility sequences and undecidable problems about rational points, J. Reine Angew. Math. 613 (2007), 1-33.

[5] S. David, Minorations de formes linéaires de logarithmes elliptiques, Mém. Soc. Math. France (N.S.) 62 (1995).

[6] M. Einsiedler, G. Everest and T. Ward, Primes in elliptic divisibility sequences, LMS J. Comput. Math. 4 (2001), 1-13.

[7] N. Elkies, Rational points with small height, www.math.harvard.edu/ elkies/ low_height.html.

[8] G. Everest and H. King, Prime powers in elliptic divisibility sequences, Math. Comp. 74 (2005), 2061-2071.

[9] G. Everest, V. Mahé and J. Reynolds, The uniform primality conjecture for congruent number curves, preprint.

[10] G. Everest, G. McLaren and T. Ward, Primitive divisors of elliptic divisibility sequences, J. Number Theory 118 (2006), 71-89.

[11] G. Everest, V. Miller and N. Stephens, Primes generated by elliptic curves, Proc. Amer. Math. Soc. 132 (2004), 955-963.

[12] M. Hindry and J. H. Silverman, The canonical height and integral points on elliptic curves, Invent. Math. 93 (1988), 419-450.

[13] —, - Diophantine Geometry, Grad. Texts in Math. 201, Springer, New York, 2000.

[14] P. Ingram, Elliptic divisibility sequences over certain curves, J. Number Theory 123 (2007), 473-486.

[15] - Multiples of integral points on elliptic curves, preprint.

[16] - A quantitative primitive divisor result for elliptic divisibility sequences, preprint.

[17] P. Ingram and J. H. Silverman, Uniform estimates for primitive divisors in elliptic divisibility sequences, preprint.

[18] S. Lang, Elliptic Curves: Diophantine Analysis, Grundlehren Math. Wiss. 231, Springer, Berlin, 1978.

[19] MAGMA, version V2.13, University of Sydney, http://magma.maths.usyd.edu.au/ magma/index.html.

[20] D. W. Masser and G. Wüstholz, Estimating isogenies on elliptic curves, Invent. Math. 100 (1990), 1-24. 
[21] B. Mazur, Modular curves and the Eisenstein ideal, Inst. Hautes Études Sci. Publ. Math. 47 (1977), 33-186.

[22] PARI-GP, http://www.parigp-home.de.

[23] J. Pesenti et L. Szpiro, Inégalité du discriminant pour les pinceaux elliptiques à réductions quelconques, Compositio Math. 120 (2000), 83-117.

[24] B. Poonen, Hilbert's Tenth Problem and Mazur's Conjecture for large subrings of $\mathbb{Q}$, J. Amer. Math. Soc. 16 (2003), 981-990.

[25] R. Shipsey, Elliptic divisibility sequences, PhD thesis, Univ. of London, 2000.

[26] J. H. Silverman, The Arithmetic of Elliptic Curves, Grad. Texts in Math. 106, Springer, New York, 1986.

[27] - Advanced Topics in the Arithmetic of Elliptic Curves, Grad. Texts in Math. 151, Springer, New York, 1994.

[28] —, A quantitative version of Siegel's theorem: integral points on elliptic curves and Catalan curves, J. Reine Angew. Math. 378 (1987), 60-100.

[29] - Wieferich's criterion and the abc-conjecture, J. Number Theory 30 (1988), $226-237$.

[30] —, Common divisors of elliptic divisibility sequences over function fields, Manuscripta Math. 114 (2004), 431-446.

[31] -, p-adic properties of division polynomials and elliptic divisibility sequences, Math. Ann. 332 (2005), 443-471, addendum, ibid., 473-474.

[32] J. H. Silverman and N. Stephens, The sign of an elliptic divisibility sequence, J. Ramanujan Math. Soc. 21 (2006), 1-17.

[33] M. Streng, Divisibility sequences for elliptic curves with complex multiplication, preprint.

[34] M. Ward, Memoir on elliptic divisibility sequences, Amer. J. Math. 70 (1948), 31-74.

[35] K. Zsigmondy, Zur Theorie der Potenzreste, Monatsh. Math. 3 (1892), 265-284.

School of Mathematics

University of East Anglia

Norwich NR4 7TJ, UK

E-mail: g.everest@uea.ac.uk

v.mahe@uea.ac.uk

shaun.stevens@uea.ac.uk
Department of Mathematics

University of Toronto

Canada M5S 2E4

E-mail: pingram@math.utoronto.ca

Received on 11.1.2008 